\title{
28 Research Suare \\ Identification of a Prognostic Six-Immune-Gene Signature and a Nomogram Model for Uveal Melanoma
}

\section{Binghua Yang}

Guangdong Second Provincial General Hospital

\section{Yuxia Fan}

Gansu Provincial Hospital

Renlong Liang

Guangdong Second Provincial General Hospital

Yi Wu

Guangdong Second Provincial General Hospital

Aiping Gu ( $\nabla$ aipinggu163@163.com )

Guangdong Second Provincial General Hospital

\section{Research Article}

Keywords: uveal melanoma, immune, prognostic signature, nomogram

Posted Date: January 5th, 2022

DOI: https://doi.org/10.21203/rs.3.rs-1148784/v1

License: (c) (i) This work is licensed under a Creative Commons Attribution 4.0 International License. Read Full License 


\section{Abstract}

Background: To identify an immune-related prognostic signature and find potential therapeutic targets for uveal melanoma.

Methods: The RNA-sequencing data obtained from The Cancer Genome Atlas (TCGA) and Gene Expression Omnibus (GEO) datasets. The prognostic six-immune-gene signature was constructed through least absolute shrinkage and selection operator and multi-variate Cox regression analyses. Functional enrichment analysis and single sample GSEA were carried out. In addition, a nomogram model established by integrating clinical variables and this signature risk score was also constructed and evaluated.

Results: We obtained 130 prognostic immune genes, and six of them were selected to construct a prognostic signature in the TCGA uveal melanoma dataset. Patients were classified into high-risk and low-risk groups according to a median risk score of this signature. High-risk group patients had poorer overall survival in comparison to the patients in the low-risk group $(p<0.001)$. These findings were further validated in two external GEO datasets. A nomogram model proved to be a good classifier for uveal melanoma by combining this signature. Both functional enrichment analysis and single sample GSEA analysis verified that this signature was truly correlated with immune system. In addition, in vitro cell experiments results demonstrated the consistent trend of our computational findings.

Conclusion: Our newly identified six-immune-gene signature and a nomogram model could be used as meaningful prognostic biomarkers, which might provide uveal melanoma patients with individualized clinical prognosis prediction and potential novel treatment targets.

\section{Background}

Uveal melanoma (UVM) is a rare eye malignant carcinoma, but in the adult population, it is the most common primary intraocular tumor [1]. Its incidence is often less than 10 per million population one year[2]. The main treatment options, including enucleation, local resection, and radiation therapies, are recommended by NCCN guideline[3]. However, the mortality rate is still rather high over the past decades[4]. In particular, about half of diagnostic UVM patients may have distant metastasis via hematogenous spreading, such as liver, which has a mean overall survival time of not more than one year[5]. Therefore, it is of great urgency to find novel useful biomarkers to predict the prognosis of UVM patients.

UVM, also known as an immune-privileged disease, displayed an unsatisfactory curative effect towards immunotherapy strategies previously[2, 6]. However, encouraging discoveries about immune therapy on UVM have been reported recently. Tebentafusp, an immune melanoma-associated-antigen (gp100)targeting anti-CD3/ TCR bispecific fusion protein, has been designed to guide T lymphocytes to kill gp100-expressing UVM cells. In 2020, a multicenter phase I/II clinical trial about Tebentafusp conducted on eighty-four metastatic UVM patients reported a promising one-year overall survival rate of $65 \%[7]$, 
which was consistent with previous reports[8]. In addition, a combination treatment of anti-angiogenic therapy with immunotherapy to cure metastatic UVM was proposed, based on the theory that targeting VEGF could not only inhibit angiogenesis, but also change the tumor microenvironment, which would make UVM cells more immune-responsive[9]. And this theory has been successfully proved in some malignant tumors, such as advanced cutaneous melanoma[10], non-small cell lung cancer[11], and advanced renal cell carcinoma[12]. Taken together, immunotherapy might demonstrate a bright future in treating UVM patients. However, few studies have systematically studied useful immune biomarkers to predict an overall survival outcome for UVM patients and find out UVM patients who might benefit from immunotherapy option.

Therefore, in the current study, we assumed that a comprehensive immune-gene signature can be a prognostic biomarker for UVM patients. We constructed and evaluated the diagnostic efficacy and predictive independence of a six-immune-gene signature in the TCGA UVM cohort, validated it in two GEO datasets, and built a prognostic nomogram model in the TCGA UVM cohort, to provide some helpful information for UVM patients prognosis and therapy.

\section{Materials And Methods}

\section{The collection of RNA-sequencing data with clinical information}

The RNA-sequencing data with clinical information were downloaded from TCGA-UVM dataset (URL: https://portal.gdc.cancer.gov/) and two GEO datasets (URL: https://www.ncbi. nlm.nih.gov/gds/? term=GSE84976, https://www.ncbi.nlm.nih.gov/gds/?term =GSE22138).

\section{Identification of prognostic immune genes in the TCGA UVM dataset}

Three hundred thirty-two immune genes were summarized from two immune-related gene sets in the Molecular Signatures Database v4.0 (URL:http://www.broadinstitute. org/gsea/msigdb/index.jsp: IMMUNE_SYSTEM_PROCESS: M13664, IMMUNE_ RESPONSE: M19817). Both univariate Cox regression and K-M survival analyses were performed. The overlapping prognostic immune genes were obtained by using Venn R package. Then, least absolute shrinkage and selection operator (LASSO) Cox regression analysis was used to select genes for the next multivariate Cox regression analysis. The corresponding coefficients were then obtained to construct the risk score formula as following: Risk score $=$ Exp_immune_gene-1 $\times$ Coef_immune_gene- $1+$ Exp_immune_gene- $2 \times$ Coef_immune_gene- $2+\ldots+$ Exp_immune_gene-n $\times$ Coef_immune_gene-n.

\section{Evaluation of the prognostic six-immune-gene signature in the TCGA UVM dataset and validation in two GEO datasets}

Based on this newly identified six-immune-gene signature, individual patients were divided into the highor low-risk group using a median cutoff value in the TCGA UVM dataset. Further analyses of survival time, status of each patient and heatmap were performed. The K-M survival analysis was performed 
using survminer and survival R packages. Time-dependent and multivariate ROC analyses were conducted to evaluate the diagnostic efficacy by using the timeROC, survivalROC, survminer, and survival $\mathrm{R}$ packages. In addition, the univariate and multivariate Cox regression analyses were utilized to evaluate the efficiency of this signature to independently predict the survival outcomes of UVM patients.

Next, and most importantly, both stability and reliability of this signature were ideally validated in two GEO datasets, including GSE84976 and GSE22138.

\section{Clinical correlation and stratification survival analysis of the prognostic six-immune-gene signature in the TCGA UVM dataset}

To further evaluate the potential clinical application of this signature, the clinicopathological variables in the TCGA UVM dataset were stratified into different subgroups accordingly. This includes age ( $<65$ and $\geq 65$ ), gender (male and female), histological subtype (single (E/S) and mixed subtype), TNM stage (stage II+III and stage IV), T stage (T 2+3 and T 4), M stage (M0 and M1), N stage (N0 and N1), new tumor event (NO and YES), and tumor basal diameter ( $<15$ and $\geq 15)$. The risk score values were compared between different subgroups by using beeswarm $\mathrm{R}$ package. The K-M survival analysis was performed by using the survminer and survival $\mathrm{R}$ packages.

\section{Functional enrichment analysis in the TCGA UVM dataset}

To further reveal the potential enriched pathway functions between high- and low-risk group patients based on this newly identified signature, the cluster Profiler $\mathrm{R}$ package was used to conduct the Gene Ontology (GO) and Kyoto Encyclopedia of Genes and Genomes (KEGG) enrichment analyses ( $p$-value < 0.05).

\section{Single sample Gene Set Enrichment Analysis (ssGSEA) in the TCGA UVM dataset}

Single sample Gene Set Enrichment Analysis (sSGSEA), as an extensive application of GSEA, it calculates an enrichment score, which represents that the genes in a particular gene set is increased or decreased in a sample. The ssGSEA was used to quantify the enrichment levels of immune cells, and immune functions between high risk and low risk groups patients.

\section{Construction and validation of a predictive nomogram model in the TCGA UVM dataset A predictive} nomogram model was constructed by combining the age, gender, histological type, TNM stage, new tumor event, tumor basal diameter, and this signature in the TCGA UVM dataset. The patients were separated into low- and high-nomogram-score groups by the median cutoff value of nomogram score. The calibration analysis was performed to explore the discrimination by using a bootstrap method. The $\mathrm{K}-\mathrm{M}$ survival and time-independent ROC analyses were also performed to evaluate the diagnostic efficacy.

\section{Cell culture and transfection}


Cells were maintained in complete DMEM medium containing with 10\% FBS (GE ${ }^{\mathrm{TM}}$ Hyclone, Utah, U.S.), $100 \mathrm{U} / \mathrm{ml}$ penicillin and $100 \mathrm{mg} / \mathrm{ml}$ streptomycin (SH30010, GE ${ }^{\text {TM }}$ Hyclone, Utah, U.S.) and cultured in a humidified atmosphere of $37^{\circ} \mathrm{C}$ and $5 \% \mathrm{CO}_{2}$.

M17 cells were subcultured one day before transfection to achieve a convergence of $30 \%-50 \%$. Lipofectamine 2000 was used for transfection with a working concentration of 50nM and OPTI-MEM medium was used for transfection. After incubation for 4 hours, the cells were replaced with cell growth medium. Cell functional cell proliferation was detected 48 hours later.

\section{Realtime PCR}

Whole cell RNA was extracted by Trizol (15596018, Life Technologies, USA) method and then RNA transcribed into cDNA using a BeyoRT ${ }^{\mathrm{TM}}$ III cDNA Synthesis Kit (Beyotime Biotechnology, Shanghai, China). BeyoFast ${ }^{\mathrm{TM}}$ SYBR Green qPCR Mix (2X) (D7260, Beyotime Biotechnology, Shanghai, China) kit were used to performed Real-time PCR assay. The qPCR experimental results were calculated by $2^{-} \triangle \triangle \mathrm{CT}$ method. Primer sequences were shown in Table 1:

\section{Western Blot}

We used BeyoLytic ${ }^{\text {TM }}$ Mammalian active protein extraction reagents (Beyotime Biotechnology, Shanghai, China) to extract the total cell protein. Then 30-40 $\mu \mathrm{g}$ total cell protein were used to perform SDSpolyacrylamide gel electrophoresis and protein transfer assay. Then the transferred NC membrane incubated with the corresponding primary antibody: Anti-CCL18 (ab233099, abcam, USA) and AntiGAPDH (ab8245, abcam, USA) (1:500) at $4^{\circ} \mathrm{C}$ overnight.

\section{CCK8 counting assay}

Digest each group cells and divide cells into 96-well plates, with $1 \times 10^{4}$ cells per well. Collect cells at each time point ( 0 h, 24 h, 48 h, 72 h) and add 10 ul CCK8 solution single solution cell proliferation detection liquid. After incubating for 4 hours, use a microplate reader to detect the absorbance of OD492.

\section{Annexin V-FITC Apoptosis Detection}

We used Annexin V-FITC Apoptosis Detection Kit (C1062S, Beyotime Biotechnology, Shanghai, China) to detect cell apoptosis, and carried out experimental operations according to Annexin V-FITC Apoptosis Detection Kit 's instructions.

\section{Cell migration and invasion assay}

In this study, we performed trans-well assay to detect cell migration and invasion followed the "In vitro Cell Migration and Invasion Assays, doi: 10.3791/51046" article instructions.

\section{Statistical analysis}


The data of our study were extracted and sorted using the PERL programming language (http://www.perl.org/, Version 5.30.0). All statistical analyses were carried out by using the R software (v 4.0.3: http://www.r-project.org). The data used in this paper are expressed as mean \pm standard error of three independent measurements. All statistics were analyzed by T-test, and the data analysis software was GraphPad Prism 6. $P<0.05$ was considered statistically significant. ${ }^{*} P<0.05 ; * \star P<0.01 ; * \star \star P<0.001$.

\section{Results}

\section{Clinical characteristics of all UVM patients}

The TCGA UVM dataset $(\mathrm{N}=80)$ was utilized to construct the prognostic immune-gene signature. GSE84976 $(\mathrm{N}=28)$ and GSE22138 $(\mathrm{N}=63)$ datasets were used as the validation cohorts. All clinical characteristics are summarized in Table 2.

\section{Identification of the prognostic six-immune-gene signature in TCGA UVM dataset}

One hundred thirty overlapping prognostic immune genes were obtained from the univariate Cox regression analysis and K-M survival analysis (Fig $1 \mathrm{~A})$. Then, 14 prognostic immune genes were selected by LASSO Cox regression analysis (Fig 1B-C). Finally, a six-immune-related-gene signature was identified (Fig 1D), whose risk score of each patient was generated using the following risk score formula: Risk score $=$ Exp_JAG2 $\times 0.157+$ Exp_CCL18 $\times 0.046+$ Exp_PRELID1 $\times 0.284+$ Exp_ CXCL8 $\times 1.154+$ Exp_PTGER4 $\times 0.092$ - Exp_ GTPBP1 $\times 0.576$ (Table 3 ). In addition, the K-M survival results of these six immune genes were presented in Fig 1E-J.

\section{Evaluation of the six-immune-gene signature in TCGA UVM dataset}

The risk score, survival time and survival status of each patient are plotted in Fig 2A-B. The heat map is displayed in Fig $2 \mathrm{C}$. The overall survival time of the low-risk group patients was significantly higher than that of the high-risk group patients (Fig 2D, $p<0.001$ ). The AUCs of the time-dependent ROC at 1-, 2- and 3 year were $0.962,0.943$, and 0.962 , respectively (Fig 2E). The AUC of multi-variate ROC for the 6 -immunegene signature risk score was 0.97 , which was much better than that of other clinical variables (Fig $2 \mathrm{~F}$ ). The PCA analysis showed that this six-immune-gene signature could help distinguish the high-risk patients from the low-risk patients ideally (Fig 2G).

Moreover, the results of univariate and multivariate Cox regression analyses of the age, gender, histological type, TNM stage, new tumor event, tumor basal diameter and this signature risk score, are shown in Fig $2 \mathrm{H}-\mathrm{I}$. Risk score of this newly identified signature $(\mathrm{HR}=2.117,95 \% \mathrm{Cl}=1.525-2.938, p<$ 0.001 ) was an independent clinical prognostic risk factor (Fig 2l).

\section{Validation of the six-immune-gene signature in the two GEO datasets}

To further confirm the predictive diagnostic power and stability of this six-immune-gene signature in predicting the overall survivals of UVM patients, we validated it in two GEO datasets, including GSE84976 
$(\mathrm{N}=28)$ (Fig 3) and GSE22138 ( $\mathrm{N}=63$ ) (Fig 4). Risk scores were also generated using the same risk score formula constructed in the TCGA UVM dataset.

The risk score, survival time and survival status of each patient in the two GEO datasets were displayed (Fig 3A-B, 4A-B). The heat map of the expression of this six-immune-gene signature was plotted (Fig 3C, $4 C)$. The overall survival time of the low-risk group patients was significantly higher than that of the highrisk group patients (Fig 3D, 4D, $p<0.001$ ). In addition, in GSE84976, the AUCs of the time-dependent ROC at $1-, 2$ - and 3 year were $0.813,0.859$, and 0.782 , respectively (Fig 2E). In GSE22138, the AUCs of the timedependent ROC at $1-, 2$ - and 3 year were $0.551,0.652$, and 0.629 , respectively (Fig 3E). The PCA analysis indicated that this six-immune-gene signature could largely help distinguish the high-risk patients from the low-risk patients (Fig 3F, 4F).

\section{Clinical correlations in the TCGA UVM dataset}

Consistent with our expectation, patients with single subtype, higher TNM stages (stage IV), higher T stage (T4), higher M stage (M1), new tumor event (YES), and tumor basal diameter ( $\geq 15)$, had significant higher risk scores than those with mixed subtype, lower TNM stages (stage II+ III), lower T stage (T2+3), M0, new tumor event (NO), and tumor basal diameter ( $<15)$ (all $p<0.05$ ) (Fig $5 \mathrm{C}-\mathrm{G}$ ). The risk score in age (Fig 5A) and gender (Fig 5B) did not show significant differences. These findings suggested that high risk score of this signature might be involved with the disease progression of UVM patients.

\section{Stratification survival analysis in the TCGA UVM dataset}

Compared with the patients in the low-risk group, those in the high-risk group had a worse outcome in many different subgroups, including age (Fig 6A-B), gender (Fig 6C-D), new tumor event (NO) (Fig 6E), tumor basal diameter (Fig 6G-H), TNM stage (Fig 6I-J), T3+4 (Fig 6K), M0 (Fig 6L), N0 (Fig 6M), mixed subtype (Fig 60), and spindle cell subtype (Fig 6P) (all $p<0.05$ ), but not in the group with new tumor event (YES) (Fig 6F), and epithelioid cell subtype (Fig 6N) (all $p>0.05)$. However, they all showed the same tendency.

\section{Functional enrichment analysis in the TCGA UVM dataset}

The functional enrichment analysis of $\mathrm{GO}$ enrichment categories, including biological process (BP), cell component (CC) and molecular function (MF), displayed the enrichment of some known immune-related pathways, including response to interferon gamma, $T$ cell activation, interferon-gamma-mediated signaling pathway, cellular response to interferon-gamma, antigen processing and presentation of peptide antigen, and so on (Fig 7A-B). Similar result was also obtained from KEGG pathway enrichment analysis (Fig 7C-D).

\section{sSGSEA analysis in the TCGA UVM dataset}

The sSGSEA indicated that the high risk patients were enriched of many immune cells, including $B$ cells, CD8+ T cells, DCs, macrophages, pDCs, Tfh, Th2 cells, TIL, and Treg, while the low risk patients were only 
enriched in aDCs (Fig 8A, all $p<0.05)$. High risk patients are enriched in all immune functions (all $p$ $<0.05$ ), except for APC-co-inhibition and Type-II IFN response (Fig 8B, all $p>0.05$ ).

\section{Construction and evaluation of the predictive nomogram model in the TCGA UVM dataset}

This newly identified predictive nomogram model was successfully constructed by combining all the details of age, gender, histological type, TNM stage, new tumor event, tumor basal diameter and this signature risk score in TCGA UVM dataset (Fig 9A). The calibration plots suggested that no significant deviations between the observed and predicted curves were found for both 1-year and 3-year survivals (Fig 9B-C). The UVM patients in high-nomogram-score group had a worse outcome compared with those in the low nomogram-score group $(p<0.001)$ (Fig 9D). The AUCs of time-dependent ROC curves for 1-, 2and 3 years were $0.977,0.980$, and 0.968 , respectively (Fig $9 \mathrm{E}$ ).

\section{Knocking-down of CCL18 expression inhibits uveal melanoma cells proliferation, migration and invasion.}

To verify our computational findings, we tested the mRNA expression level of CCL18, CXCL8, GTPBP1, JAG2, PRELID1 and PTGER4 in uveal melanoma cell lines: M17, M23 and SP6.5 and normal uveal epithelial cell: Um95. We found that compared with Um95, the expression patterns of these six genes in uveal melanoma cell lines: are consistent with our computational findings (Fig 10A). In addition, we found that CCL18 has the highest expression in the M17 cell line, so we selected the CCL18 gene to perform related functional verification in M17 cells. As shown in Figure 10B\&C: siRNA-2 successfully knocked down the expression of CCL18 in M17 cells. The results of cell proliferation experiments show that low CCL18 expression can significantly inhibit the proliferation ability of M17 cells (Fig 10D). Inhibition of CCL18 expression will significantly inhibit the migration and invasion of M17 cells $\otimes F i g ~ 11$ A\&BQDFurthermore, knocking down the expression of CCL18 significantly induced M17 cell apoptosis (Fig $11 \mathrm{C}$ ). In summary: Our results demonstrated that our computational findings were consistent with the trend of in vitro cell experiments.

\section{Discussion}

In this study, we identified 130 prognostic immune genes that strictly met the criteria of both univariate Cox regression analysis and K-M survival analysis, then used LASSO and multivariate Cox regression analyses to ultimately generate six immune-genes to construct an immune-related signature for predicting the prognosis of UVM patients. We found that the overall survival time was shorter of patients in the high-risk group than those in the low-risk group. Moreover, taken together with the results of timedependent and multivariate ROC analysis, it showed a satisfactory diagnostic efficacy. In addition, the predictive independence was also confirmed. Most importantly, these findings were validated in two external GEO datasets.

Previous studies have reported some clinical variables that may affect the prognosis of UVM patients, including fair skin, light-colored eyes, congenital ocular melanocytosis, karnofsky index, largest dimension of the largest metastasis site, metastatic burden, serum transaminase, lactate dehydrogenase, 
and alkaline phosphatase level $[2,13,14]$. These clinical factors may really help clinical doctors offer optimal treatment and make a good prognosis prediction for UVM patients. However, these variables had not been recorded in the TCGA dataset. Therefore, instead, we included some typical variables in the dataset, such as the histological subtype, clinical stage, new tumor event, tumor basal diameter, tumor thickness, and some traditional demographical indexes, including age, and gender. Surprisingly, we came out that only age and our immune-related signature were found as independent risk factors. Age as an independent risk factor was also identified on a metastatic UVM research[15], but it was not consistent with another immune-related signature published study previously [16].

Compared with the previously published immune biomarker study by Li et.al[16], we did make some progresses on some aspects. First, half of these six-immune-genes we selected had been validated to be correlated with UVM functions in vitro, while none of the previous study. For example, JAG2 promoted UVM cells growth and metastasis[17]. Besides, inflammation-induced CXCL8 might stimulate the UVM cells chemotactic capacity[18]. CCL18 could enhance UVM cell line growth through coculture with human retinal pericytes[19]. In addition, the function of the left three immune genes were also cancer-related[2022]. Second, in our study, the AUC values of time-dependent ROC were higher in both 1 - and 3 year $(0.962$ vs $0.82,0.962$ vs 0.94 , respectively), which demonstrated a better diagnostic efficacy. Moreover, we also performed a multivariate ROC analysis, which directly showed that our immune-related signature was better than any other clinical variables. Third, our newly identified immune-related signature was also successfully validated in two external GEO datasets, which suggested a potential clinical application. Fourth, and importantly, we first constructed an immune-related nomogram model combining multiple clinical variables, together with this signature risk score in the UVM dataset cohort. The result of calibration analyses demonstrated a good consistence between the predicted and actual curves. Taken together with the K-M survival analysis and ROC curves, this prognostic six-immune-gene signature can accurately predict the OS of UVM patients and exhibit great potential for clinical applications, including individualized prognosis and therapy.

To further confirm that the enrichment function of this signature is truly correlated with immune function, first, we did a functional enrichment analysis on the differently expressed genes between the high-risk and low-risk groups, and the result demonstrated an enrichment of immune-related pathways, including response to interferon gamma, $T$ cell activation, interferon-gamma-mediated signaling pathway, cellular response to interferon-gamma, antigen processing and presentation of peptide antigen, which actually successfully supported our findings. Second, we also performed the sSGSEA to evaluate the enriched types of immune cells and functions in high- and low-risk group patients, and it came out that high risk patients were enriched of many immune cells, including B cells, CD8+ T cells, DCs, macrophages, pDCs, Tfh, Th2 cells, TIL, and Treg, while low risk patients only enriched in aDCs. Moreover, high risk patients were enriched of all immune functions, except for APC-co-inhibition and Type-II IFN response. Taken these two kinds of data together, we believed that this newly identified signature as a biomarker is useful to predict the prognosis on the immune therapy. 
There are some limitations in our current study. First, these findings were obtained through mRNA level in public databases, so the following validations on protein expression level, in-vivo and clinical sample are needed. Second, external validation on patients treated with immunotherapy is also needed to further confirm the application of our signature and nomogram model. Therefore, we will continue to conduct an in-depth study to illustrate the molecular mechanisms of six immune genes, and make this signature and nomogram model more convincing for clinical application in the future.

\section{Conclusions}

We successfully constructed a prognostic six-immune-gene signature using public TCGA UVM dataset and validated it in two GEO datasets. This signature was confirmed to have promising diagnostic and predictive efficacies as a biomarker. In addition, the novel nomogram model was confirmed as a good predictive biomarker. These findings could provide UVM patients with individualized clinical prognostic prediction and potential novel treatment targets.

\section{Abbreviations}

TCGA: The Cancer Genome Atlas; GEO: Gene Expression Omnibus; UVM: Uveal melanoma; LASSO: least absolute shrinkage and selection operator; GO: Gene Ontology; KEGG: Kyoto Encyclopedia of Genes and Genomes; ssGSEA: Single sample Gene Set Enrichment Analysis; BP: biological process; CC: cell component; MF: molecular function;

\section{Declarations}

\section{Acknowledgements}

Not applicable.

\section{Authors' contributions}

A.G. designed the experiments and review the article. B.Y., Y.F., Y.W. and R.L. devoted to the data extraction, analysis tools, and statistical analysis. B.Y. and Y.F. did data extraction, analyze the data, interpreted the results and wrote the article. All authors have read and approved the manuscript

\section{Funding}

Not applicable.

\section{Availability of data and materials}

All data of this study are available in TCGA-UVM dataset (URL: https://portal.gdc.cancer.gov/) and two GEO datasets (URL: https://www.ncbi.nlm.nih.gov/gds/?term=GSE84976, https://www.ncbi.nlm.nih.gov/gds/?term =GSE22138). 


\section{Ethics approval and consent to participate}

This article does not contain any studies with human participants or animals performed by any of the authors. All methods were performed in accordance with the relevant guidelines and regulations.

\section{Consent for publication}

Not applicable.

\section{Competing interests}

The authors all declare no competing interests.

\section{References}

1. Kaliki S, Shields CL. Uveal melanoma: relatively rare but deadly cancer. Eye (Lond). Feb 2017;31(2):241-257. doi: 10.1038/eye.2016.275.

2. Jager MJ, Shields CL, Cebulla CM, et al. Uveal melanoma. Nat Rev Dis Primers. Apr 9 2020;6(1):24. doi: 10.1038/s41572-020-0158-0.

3. Rao PK, Barker C, Coit DG, et al. NCCN Guidelines Insights: Uveal Melanoma, Version 1.2019. J Nat/ Compr Canc Netw. Feb 2020;18(2):120-131. doi: 10.6004/jnccn.2020.0007.

4. Spagnolo F, Picasso V, Spano L, Tanda E, Venzano C, Queirolo P. Update on Metastatic Uveal Melanoma: Progress and Challenges. BioDrugs. Jun 2016;30(3):161-172. doi: 10.1007/s40259-0160167-4.

5. Qin Y, Bollin K, de Macedo MP, et al. Immune profiling of uveal melanoma identifies a potential signature associated with response to immunotherapy. J Immunother Cancer. Nov 2020;8(2). doi: 10.1136/jitc-2020-000960.

6. Najjar YG, Navrazhina K, Ding F, et al. Ipilimumab plus nivolumab for patients with metastatic uveal melanoma: a multicenter, retrospective study. J Immunother Cancer. Jun 2020;8(1). doi: 10.1136/jitc2019-000331.

7. Middleton MR, McAlpine C, Woodcock VK, et al. Tebentafusp, A TCR/Anti-CD3 Bispecific Fusion Protein Targeting gp100, Potently Activated Antitumor Immune Responses in Patients with Metastatic Melanoma. Clin Cancer Res. Nov 15 2020;26(22):5869-5878. doi: 10.1158/10780432.Ccr-20-1247.

8. Damato BE, Dukes J, Goodall H, Carvajal RD. Tebentafusp: T Cell Redirection for the Treatment of Metastatic Uveal Melanoma. Cancers (Basel). Jul 11 2019;11(7). doi: 10.3390/cancers11070971.

9. Castet F, Garcia-Mulero S, Sanz-Pamplona R, et al. Uveal Melanoma, Angiogenesis and Immunotherapy, Is There Any Hope? Cancers (Basel). Jun 17 2019;11(6). doi: 10.3390/cancers 11060834 . 
10. Hodi FS, Lawrence D, Lezcano $C$, et al. Bevacizumab plus ipilimumab in patients with metastatic melanoma. Cancer Immunol Res. Jul 2014;2(7):632-642. doi: 10.1158/2326-6066.Cir-14-0053.

11. Reck M, Mok TSK, Nishio M, et al. Atezolizumab plus bevacizumab and chemotherapy in non-smallcell lung cancer (IMpower150): key subgroup analyses of patients with EGFR mutations or baseline liver metastases in a randomised, open-label phase 3 trial. Lancet Respir Med. May 2019;7(5):387401. doi: 10.1016/s2213-2600(19)30084-0.

12. Powles $T$, Plimack ER, Soulières $D$, et al. Pembrolizumab plus axitinib versus sunitinib monotherapy as first-line treatment of advanced renal cell carcinoma (KEYNOTE-426): extended follow-up from a randomised, open-label, phase 3 trial. Lancet Oncol. Dec 2020;21(12):1563-1573. doi: 10.1016/s1470-2045(20)30436-8.

13. Eskelin S, Pyrhönen S, Hahka-Kemppinen M, Tuomaala S, Kivelä T. A prognostic model and staging for metastatic uveal melanoma. Cancer. Jan 15 2003;97(2):465-475. doi: 10.1002/cncr.11113.

14. Kim JH, Shin SJ, Heo SJ, et al. Prognoses and Clinical Outcomes of Primary and Recurrent Uveal Melanoma. Cancer Res Treat. Oct 2018;50(4):1238-1251. doi: 10.4143/crt.2017.534.

15. Lorenzo D, Piulats JM, Ochoa M, et al. Clinical predictors of survival in metastatic uveal melanoma. Jpn J Ophthalmol. Mar 2019;63(2):197-209. doi: 10.1007/s10384-019-00656-9.

16. Li YZ, Huang Y, Deng XY, Tu CS. Identification of an immune-related signature for the prognosis of uveal melanoma. Int J Ophthalmol. 2020;13(3):458-465. doi: 10.18240/ijo.2020.03.14.

17. Asnaghi L, Handa JT, Merbs SL, Harbour JW, Eberhart CG. A role for Jag2 in promoting uveal melanoma dissemination and growth. Invest Ophthalmol Vis Sci. Jan 9 2013;54(1):295-306. doi: 10.1167/iovs.12-10209.

18. Jehs T, Faber C, Juel HB, Bronkhorst IH, Jager MJ, Nissen MH. Inflammation-induced chemokine expression in uveal melanoma cell lines stimulates monocyte chemotaxis. Invest Ophthalmol Vis Sci. Jul 29 2014;55(8):5169-5175. doi: 10.1167/iovs.14-14394.

19. Anfuso CD, Longo A, Distefano A, et al. Uveal Melanoma Cells Elicit Retinal Pericyte Phenotypical and Biochemical Changes in an in Vitro Model of Coculture. Int J Mol Sci. Aug 3 2020;21(15). doi: 10.3390/ijms21155557.

20. Gillen AE, Brechbuhl HM, Yamamoto TM, et al. Alternative Polyadenylation of PRELID1 Regulates Mitochondrial ROS Signaling and Cancer Outcomes. Mol Cancer Res. Dec 2017;15(12):1741-1751. doi: 10.1158/1541-7786.Mcr-17-0010.

21. Roulis M, Kaklamanos A, Schernthanner M, et al. Paracrine orchestration of intestinal tumorigenesis by a mesenchymal niche. Nature. Apr 2020;580(7804):524-529. doi: 10.1038/s41586-020-2166-3.

22. Huang W, Huang H, Zhang S, et al. A Novel Diagnosis Method Based on Methylation Analysis of SHOX2 and Serum Biomarker for Early Stage Lung Cancer. Cancer Control. Jan-Dec 2020;27(1):1073274820969703. doi: 10.1177/1073274820969703.

\section{Tables}


Table 1 Primer sequences for qPCR

\begin{tabular}{ll} 
Gene & Primer sequences \\
\hline CCL18 & F: GTTGACTATTCTGAAACCAGCCC \\
\hline R: GTCGCTGATGTATTTCTGGACCC \\
\hline F: GAGAGTGATTGAGAGTGGACCAC \\
\hline RTPBP1 & F: CCTTCATCGACTTGGCTGGTCA \\
\hline & R: CCAGGTGTTCTTTGGTCATCCC \\
\hline JAG2 & F: GCTGCTACGACCTGGTCAATGA \\
\hline & R: AGGTGTAGGCATCGCACTGGAA \\
\hline PRELID1 & F: GGAGGACTCTATTGTGGACCCA \\
\hline & R: CAGTCCAGCCACTGTTGTCAGA \\
\hline PTGER4 & F: TACTCATTGCCACCTCCCTGGT \\
\hline & R: GACTTCTCGCTCCAAACTTGGC \\
\hline GAPDH & F: GTCTCCTCTGACTTCAACAGCG \\
\hline
\end{tabular}

Table 2 Clinical characteristics of included uveal melanoma patients in the TCGA dataset and two GEO datasets (GSE84976 and GSE22138) 


\begin{tabular}{|c|c|c|c|}
\hline Characteristics & $\begin{array}{l}\text { TCGA UVM } \\
(\mathrm{N}=80)\end{array}$ & $\begin{array}{l}\text { GSE84976 } \\
(\mathrm{N}=28)\end{array}$ & $\begin{array}{l}\text { GSE22138 } \\
(\mathrm{N}=63)\end{array}$ \\
\hline \multicolumn{4}{|c|}{ Age at diagnosis (years) } \\
\hline$<65$ & $45(56.25 \%)$ & $12(42.86 \%)$ & $36(57.14 \%)$ \\
\hline$\geq 65$ & $35(43.75 \%)$ & $16(57.14 \%)$ & $27(42.86 \%)$ \\
\hline \multicolumn{4}{|l|}{ Sex } \\
\hline Male & $45(56.25 \%)$ & - & $40(63.49 \%)$ \\
\hline Female & $35(43.75 \%)$ & - & $23(36.51 \%)$ \\
\hline \multicolumn{4}{|c|}{ Histological subtype } \\
\hline Spindle Cell & $30(37.50 \%)$ & - & - \\
\hline Epithelioid Cell & $13(16.25 \%)$ & - & $21(33.33 \%)$ \\
\hline Mixed & $37(46.25 \%)$ & - & $23(36.51 \%)$ \\
\hline NA & - & - & $19(30.16 \%))$ \\
\hline \multicolumn{4}{|l|}{ TNM stage } \\
\hline Stage I & $0(0.00 \%)$ & - & - \\
\hline Stage II & $36(45.00 \%)$ & - & - \\
\hline Stage III & $40(50.00 \%)$ & - & - \\
\hline Stage IV & $4(5.00 \%)$ & - & - \\
\hline \multicolumn{4}{|c|}{ New tumor event } \\
\hline NO & $60(75.00 \%)$ & - & - \\
\hline YES & $20(25.00 \%)$ & - & - \\
\hline \multicolumn{4}{|c|}{ Tumor basal diameter } \\
\hline$<15$ & $20(25.00 \%)$ & - & - \\
\hline$\geq 15$ & $60(75.00 \%)$ & - & - \\
\hline
\end{tabular}

Mixed: Spindle Cell | Epithelioid Cell, and Epithelioid Cell | Spindle Cell.

Table 3. The six-immune-related signature identified from multivariate Cox analysis. 
Multivariate Cox regression analysis

\begin{tabular}{llllll}
$\begin{array}{l}\text { Gene } \\
\text { symbol }\end{array}$ & Description & Coefficient & HR & $95 \% \mathrm{Cl}$ & $p$-value \\
\hline JAG2 & Jagged2 & 0.157 & 1.170 & $1.048-1.307$ & 0.005 \\
\hline CCL18 & Chemokine 18 & 0.046 & 1.047 & $1.008-1.087$ & 0.016 \\
\hline PRELID1 & $\begin{array}{l}\text { PRELI domain-containing protein } \\
1\end{array}$ & 0.284 & 1.328 & $1.135-1.555$ & $<0.001$ \\
\hline CXCL8 & Interleukin 8 & 1.154 & 3.172 & $1.427-7.053$ & 0.005 \\
\hline PTGER4 & Prostaglandin E (2) receptor & 0.092 & 1.096 & $1.047-1.148$ & $<0.001$ \\
\hline GTPBP1 & GTP-binding protein 1 & -0.576 & 0.562 & $0.425-0.743$ & $<0.001$ \\
\hline
\end{tabular}

\section{Figures}

\section{Figure 1}

\section{Identification of the six-immune-related gene signature in TCGA UVM dataset}

(A) Venn plot of overlapping results of univariate Cox regression analysis and KM survival analysis; (B) Cvfit plot of LASSO cox regression analysis; (C) Lambda plot of LASSO Cox regression analysis; (D) Hazard ratio of the six-immune-related gene signature in multivariate Cox regression analysis; K-M survival analysis of the six selective immune-related genes: (E) JAG2; (F) CCL18; (G) PRELID1; (H) CXCL8; (I) PTGER4; (J) GTPBP1.

\section{Figure 2}

\section{Evaluation of the six-immune-related gene signature in TCGA UVM dataset}

(A-C) Distribution of risk score, survival status, and expression of each patient; (D) K-M survival curve of the high-risk and low-risk groups; (E) The time-dependent receiver operating characteristic (ROC) curves and area under the curve (AUC) at 1-, 2-, and 3 years; (F) ROC curve analysis showed the prognostic accuracy of clinicopathological parameters and the signature risk score; $(G)$ Principal components analysis (PCA) of the six-immune-related gene signature; $(\mathrm{H})$ Forest plot for univariate Cox regression analysis; (I) Forest plot for multivariate Cox regression analysis. 


\section{Figure 3}

\section{Validation of the six-immune-related gene signature in GSE84976 dataset}

(A-C) Distribution of risk score, survival status, and expression of each patient; (D) K-M survival curve of the high-risk and low-risk groups patients; (E) Time-dependent ROC curves and AUC at 1-, 2-, and 3 years; (F) PCA of the six-immune-related gene signature.

\section{Figure 4}

\section{Validation of the six-immune-related gene signature in GSE22138 dataset}

(A-C) Distribution of risk score, survival status, and expression of each patient; (D) K-M survival curve of the high-risk and low-risk groups patients; (E) Time-dependent ROC curves and AUC at 1-, 2-, and 3 years; (F) PCA of the six-immune-related gene signature.

\section{Figure 5}

\section{Clinical correlations in the TCGA UVM dataset}

The risk score distributions between the signature risk scores and the clinicopathological features in different subgroups: $(A)$ age ( $\geq 65$ vs $<65 ; p=0.412$ ); (B) gender (female vs male; $p=0.354$ ); (C) histological subtypes (single vs mixed subtype; $p=0.041$ ) (single: spindle cell subtype and epithelioid cell subtype; mixed: Epithelioid Cell | Spindle Cell, and Spindle Cell | Epithelioid Cell); (D) TNM stage (stage

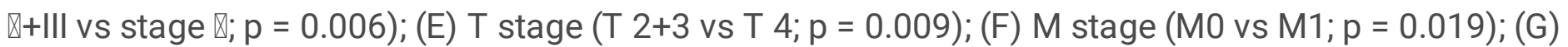
new tumor event (NTE, NO vs YES, $p=8.317 \mathrm{e}-07)$; $(\mathrm{H})$ tumor basal diameter (TBD, $\geq 15$ vs $<15, p=$ $0.004)$.

\section{Figure 6}

\section{Stratification survival analysis in the TCGA UVM dataset}

K-M survival analysis showed the overall survival time of the high- and low-risk UVM patients stratified by different variables: age (A-B); sex (C-D); new tumor event (NTE, E-F); tumor basal diameter (TBD, G-H); TNM stage (I-J), T stage (K), N stage (L), M stage (M), E: epithelioid cell subtype (N); Mixed: Epithelioid Cell I Spindle Cell, and Spindle Cell Epithelioid Cell (O); S: spindle cell subtype (P). 


\section{Figure 7}

Functional enrichment analysis of six-immune-related gene signature in the TCGA

\section{UVM dataset}

The bar plot and dot plot of Gene Ontology (GO) (A-B) and Kyoto Encyclopedia of Genes and

Genomes (KEGG) pathway (C-D) functional enrichment analyses. BP: Biological process; CC: Cellular Component; MF: Molecular function.

A

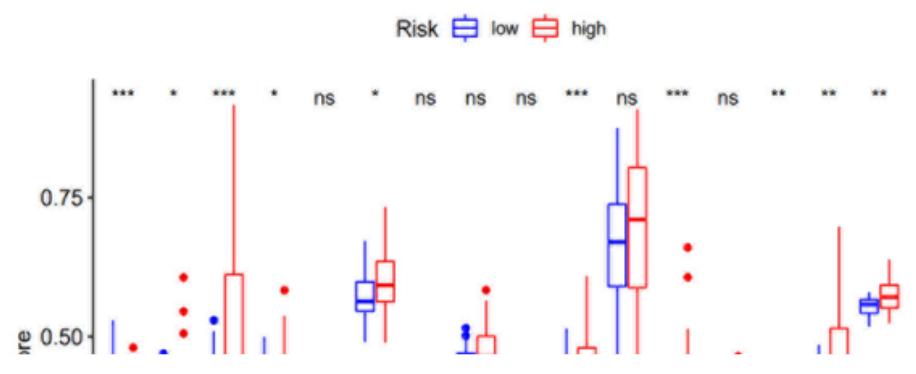

B

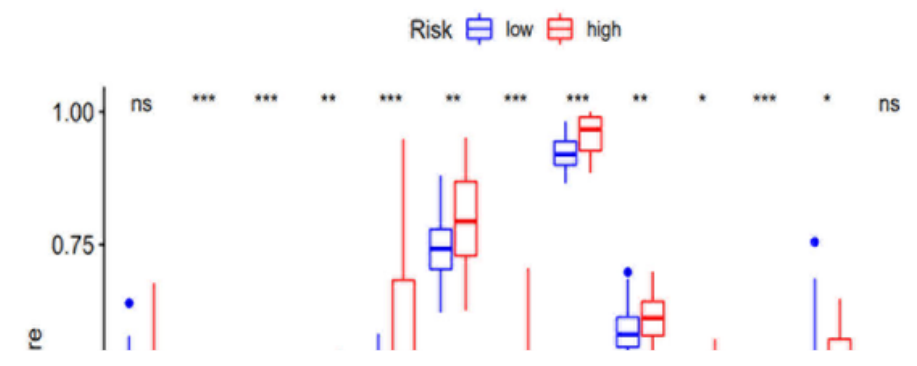

\section{Figure 8}

\section{Single-sample GSEA (ssGSEA) in the TCGA UVM dataset}

(A) The enrichment score of immune cells in the high-risk and low-risk group patients in SSGSEA; (B) The enrichment score of immune functions in the high-risk and low-risk group patients in SSGSEA. 
A

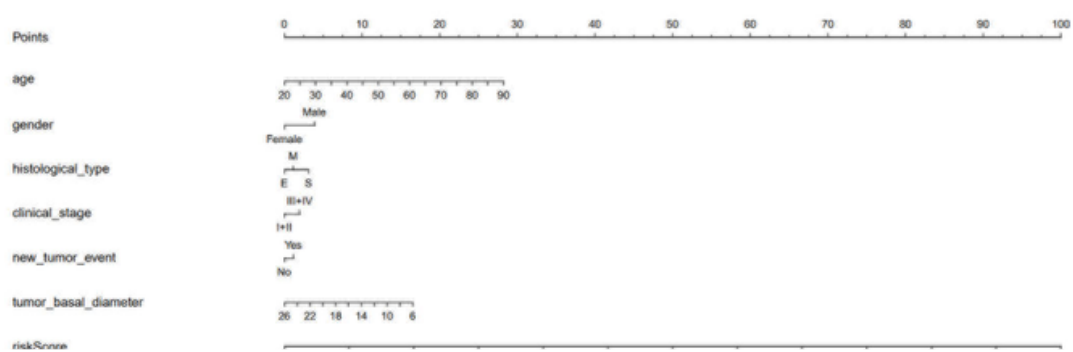

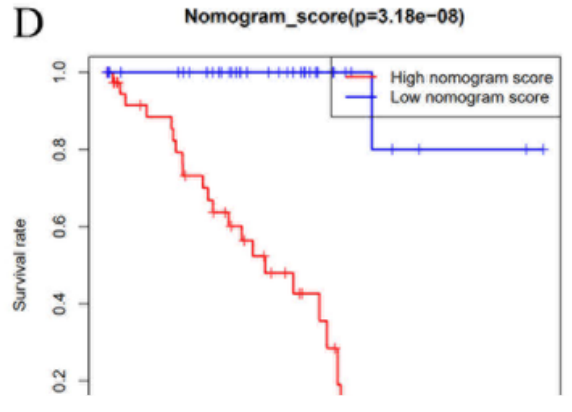

\section{Figure 9}

\section{Construction and evaluation of the prognostic nomogram model in the UVM dataset}

(A) The nomogram model was constructed by age, gender, histological type, TMN stage, new

tumor event, tumor basal diameter and six-immune-related prognostic signature risk score; (B-C) The calibration plot of the nomogram; (D) K-M survival curve between high-nomogram-score and lownomogram-score groups; (E) The AUCs of the time-dependent ROC curves at 1-, 2-, and 3 years. 
A B
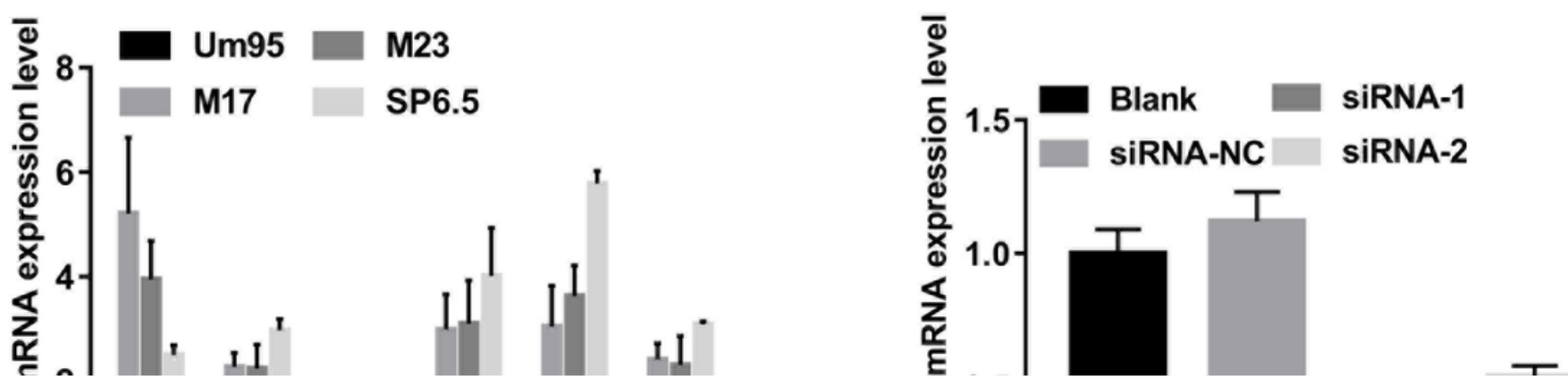

Figure 10

Knocking down of CCL18 significantly inhibits the proliferation of M17 cells

(A) qPCR experimental results of CCL18, CXCL8, GTPBP1, JAG2, PRELID1 and PTGER4 in Um95, M17, M23 and SP6.5 cells; (B) CCL18 mRNA expression level of M17 cells (blank) and siRAN-NC, siRNA-1 and siRNA-2 treated M17 cells; (C) CCL18 protein expression level of M17 cells (blank) and siRAN-NC, siRNA-1 and siRNA-2 treated M17 cells; D: CCK8 counting results of M17 cells (blank) and siRAN-NC and siRNA-1 treated M17 cells.

Figure 11 


\section{Knockdown of CCL18 inhibits M17 migration and invasion and induces M17 cell apoptosis}

A: Trans-well test results of M17 cells (blank) and siRAN-NC and siRNA-1 treated M17 cells migration (upper) and invasion (lower) capacity; B: Data statistics of Fig A; C: Flow cytometric cell apoptosis detection of M17 cells (blank) and siRAN-NC and siRNA-1 treated M17 cells. 